\title{
À propos des auteurs
}

BARBIER Jean-Marie, est professeur émérite au Conservatoire National des Arts et Métiers à Paris. Il est spécialiste de formation des adultes, de d'analyse des activités et de la construction de l'expérience. Il privilégie l'« entrée activité » en recherche, en formation et dans l'action professionnelle. Il est DHC de l'UCL (Louvain) et responsable avec Augustin Mutuale de la chaire Unesco-ICP 'Formation Professionnelle, Construction Personnelle, Transformations Sociales'. Il préside la Biennale Internationale de l'Education, de la Formation et des Pratiques Professionnelles.

DUTOIT Martine, a été enseignante-chercheuse à l'Université d'Evry, et l'est actuellement à l'Institut Catholique de Paris où ses recherches relient activités et construction de l'expérience des publics-cibles d'action sociale et/ou de santé, et activités et expérience des professionnelles qui les accompagnent. Elle s'inscrit dans une approche à dominante constructiviste et privilégie l'entrée activité. Elle est responsable du développement de la Chaire Unesco 'Formation Professionnelle, Construction Personnelle, Transformations Sociales', qui conduit des recherches collaboratives avec les professionnelles des métiers de l'humain.

GAJEK Katarzyna, pédagogue sociale, docteure en sciences humaines dans le domaine de la pédagogie, professeure adjointe à la Chaire de Pédagogie sociale et de resocialisation, de l'Université de Lodz. Ses intérêts de recherche portent sur : Gender Studies, phénomène de la violence (violence domestique, bullying, harcèlement, traite des êtres humains), phénomène d'exclusion sociale et processus d'empowerment, recherche qualitatives (en particulier la recherche biographique et l'analyse critique du discours). L'auteure de la monographie: Doświadczenie przemocy $w$ rodzinie. Autobiograficzne narracje kobiet [Expérience de la violence domestique. Récits autobiographiques de femmes] (2019).

HYPPOLITE Marie Jasmine, docteure en formation des adultes, se passionne pour les sciences sociales. Née à l'île de la Réunion (département français dans l'Océan Indien). Ses études en sciences économiques, politiques, juridiques et de l'éducation l'amènent à des postes d'enseignant dans le secondaire et le supérieur. Formatrice d'adultes elle se questionne sur la façon dont les acteurs se préparent à une négociation, ouvrant un nouveau champ de recherche : «se préparer à l'action ». 
KAFAR Marcin, chercheur interdisciplinaire travaillant à l'intersection des sciences humaines et sociales; trouve des ressources dans les champs d'anthropologie culturelle, de l'éducation, de la littéraire et de la philosophie. Il est rédacteur en chef de la série " Perspektywy Biograficzne » [Perspectives Biographiques] à l'Edition de l'Université de Lodz. Il a écrit et rédigé une dizaine de monographies, et dernièrement (avec Anna Kacperczak) Autoetnograficzne "zbliżenia» $i$ «oddalenia ». $O$ autoetnografii w Polsce [ Rapprochements » et " éloignements » auto-ethnographiques. Sur l'auto-ethnographie en Pologne], (2020). Stagiaire $(2010,2016)$ et visiting professor (2018) au département de Communication, à l'University of South Florida.

KAMIŃSKA-JATCZAK Izabela, pédagogue sociale, docteure en sciences sociales dans le domaine de la pédagogie, professeure adjointe à la Chaire de Pédagogie sociale et de resocialisation, de l'Université de Lodz. Ses intérêts de recherche portent sur : l'analyse de l'activité dans le champ de travail social, le travail social avec la famille, les contextes biographies et historiques de la pédagogie sociale, la méthodologie des recherches interprétatives. L'auteure de la monographie Aktywność asystentów rodziny. Analiza narracji w ujęciu transwersalnym [Activité des assistants familiaux. Analyse transversale des récits] (2021).

LÉONARD Myriam, docteure en sciences de l'éducation, spécialité formation des adultes, membre du laboratoire de recherche FOAP (Formation et apprentissages professionnels) au Conservatoire National des Arts et Métiers à Paris et chercheur bénévole auprès de la Chaire Unesco ICP, dirigée par Jean-Marie Barbier et Augustin Mutuale. Elle exerce en tant que formatrice de formateur et ingénieur formation dans le secteur privé, et en tant qu'enseignante à l'université auprès de futurs enseignants. Elle est également fondatrice d'une entreprise qui promeut la recherche comme outil de formation des professionnels et acteurs du champ de la formation professionnelle en France. Elle a passé six années auprès de policiers, en tant que chercheure dans un premier temps, puis en tant que Chef de la section Recherche, Etudes et Innovation de la direction de la formation de la Police nationale française.

MARYNOWICZ-HETKA Ewa, pédagogue sociale, professeure en sciences humaines, professeure émérite de l'Université de Lodz, Docteur Honoris Causa de l'Université d'Ostrava. Intérêts de recherche: réflexion sur les fondements de l'action du pédagogue social dans le champ de la pratique ; formation aux professions sociales et à l'action dans le champ du travail social; prémisses épistémologiques et méthodologiques de construction des outils d'analyse d'activité dans le champ de la pratique. Rédactrice en chef de la revue "Sciences de l'éducation. Les études interdisciplinaires » portée par la Faculté de Sciences de l'éducation l'Université de Lodz et Maison d'édition de l'Université de Lodz. 
RODRIGUEZ Daniela, docteure en formation des adultes et chercheuse associée au Centre des recherches sur le travail et le développement, équipe Psychosociologie du travail et de la formation, anthropologie des pratiques du Conservatoire National des Arts et Métiers à Paris. Ses travaux de recherche portent sur les liens entre travail et formation à l'échelle de la biographie professionnelle des sujets.

VU Kim, docteure en sciences de l'éducation, membre du laboratoire de recherche FOAP (Formation et apprentissages professionnels) au Conservatoire National des Arts et Métiers à Paris. Sa thèse, soutenue le 16 juin 2020 sous la direction de Jean-Marie Barbier et Line Numa-Bocage part de l'hypothèse selon laquelle à travers l'expérience, le manager peut modifier sa façon de faire agir les collaborateurs, et ainsi se doter de compétences humaines pour exercer son activité de manager.

WALCZAK Anna, docteure habilitée, professeure de l'Université de Lodz, travaille à la Chaire de Pédagogie sociale et de resocialisation, à la Faculté des Sciences de l'Education de l'UL. Ses intérêts de recherche portent sur l'anthropologie philosophique, la philosophie de l'éducation et de l'éthique du point de vue phénoménologique - herméneutique et discursif. Dans ses travaux, elle procède à la (re)construction de la pédagogie existentielle en faisant appel à la narratologie ainsi qu'aux approches biographiques (l'identité narrative), à l'hétérologie, à la psychologie de la culture inspirée principalement par la pensée de C. G. Jung.

WITKOWSKI Lech, professeur en sciences humaines, philosophe et pédagogue, a publié 20 livres, a été (co)rédacteur de 15 publications, maître de conférence à l'Académie de Poméranie à Slupsk, il s'intéresse à la philosophie de l'éducation et à l'histoire de la pensée pédagogique, construit une perspective dite de « humanisme appliqué », ses études monographiques sont consacrées aux catégories suivantes : l'autorité, le cycle de vie, la dualité, l'identité, il reste sous l'influence des conceptions des auteurs comme: Bachelard, Bachtin, Bauman, Erikson, Giroux, Habermas, Radlińska.

WRÓBLEWSKA Halina Monika, docteure habilitée, professeure de l'Université à Bialystok, à la Faculté des Sciences de l'Education. Ses intérêts de recherche concernent les problèmes suivants: les compétences créatives du sujet et les conditions pour leur développement; le diagnostic, la formation et le soutien au développement des capacités créatives et des compétences dans le processus de l'éducation; les conditions de développement d'un élève doué ; l'éducation de soutien au développement de l'activité créative ; l'art et l'expression créative dans le développement et le soutien de l'auto développement créatif ; l'expérience créative dans les narrations et récits biographiques. L'auteure et rédactrice de nombreuses publications, entre autres : Kompetencje twórcze $w$ dorosłości [Compétences créatives à l'âge adulte] (2015) ; Potencjał podmiotowy i kompetencje - w perspektywie rozwoju kariery zawodowej [Le potentiel du sujet et ses compétences - dans la perspective du développement de la carrière professionnelle] (éd.) (2018). 